DR. MARTA SCARIONI (Orcid ID : 0000-0002-2420-6447)

Article type : Short Communications

\title{
Late-onset presentation and phenotypic heterogeneity of the rare R377W PSEN1 mutation
}

Marta Scarioni ${ }^{1,2,3,4^{*}}$, Andrea Arighi ${ }^{1,2}$, Chiara Fenoglio ${ }^{1,2}$, Federica Sorrentino ${ }^{1,2}$, Maria Serpente $^{1,2}$, Emanuela Rotondo ${ }^{1,2}$, Matteo Mercurio ${ }^{1,2}$, Giorgio Marotta ${ }^{1}$, Anke A. Dijkstra ${ }^{3}$, Yolande A.L. Pijnenburg ${ }^{4}$, Elio Scarpini ${ }^{1,2}$, Daniela Galimberti ${ }^{1,2}$

1. Fondazione IRCCS Ca' Granda Ospedale Maggiore Policlinico, Via F. Sforza 35, 20122, Milan, Italy

2. University of Milan, Dino Ferrari Center, Milan, Italy

3. Department of Pathology, Amsterdam University Medical Centers, location Vumc, Amsterdam Neuroscience, Amsterdam, The Netherlands

4. Department of Neurology, Amsterdam University Medical Centers, location VUmc, Alzheimer Center, Amsterdam, The Netherlands

*Corresponding author: Marta Scarioni

Email: m.scarioni@amsterdamumc.nl - tel: +31204440685 - fax: +31204444586

Running title: Late-onset AD and R377W PSEN1 mutation

Abstract word count: 170. Main text word count: 1499. Total word count: 2323.

This article has been accepted for publication and undergone full peer review but has not been through the copyediting, typesetting, pagination and proofreading process, which may lead to differences between this version and the Version of Record. Please cite this article as doi: 10.1111/ENE.14506

This article is protected by copyright. All rights reserved 
Keywords: 1) Presenilin 1 2) Mutation 3) Alzheimer's disease 4) Late Onset Alzheimer Disease 5) Frontotemporal Dementia

Disclosure: none.

This article is protected by copyright. All rights reserved 


\section{Abstract}

Background: Mutations in the PSENI gene are the most common cause of autosomal-dominant Alzheimer's disease and have been associated with the earliest disease onset. We describe an unusual presentation of the rare R377W PSEN1 mutation with a late age of onset, and we provide for the first time in vivo pathological evidence for this mutation. Methods: A 71-years old female patient with progressive cognitive decline in the past three years and positive family history for dementia underwent neurological evaluation, neuropsychological testing, lumbar puncture, conventional brain imaging, amyloid-PET and extensive genetic screening with a next generation sequencing technique. Results: The diagnostic work-up revealed mixed behavioural and amnestic disease features on neuropsychological tests, MRI, and FDG-PET. Amyloid-PET detected amyloid deposition in the frontal areas, in the parietal lobes and the precunei. The genetic screening unraveled the presence of the rare $R 377 W$ mutation in the PSEN1 gene. Conclusions: Extensive genetic screening is advisable also for late-onset presentations of Alzheimer's disease, especially in the presence of a positive family history or atypical clinical features. 


\section{Introduction}

Since cerebrospinal fluid (CSF) biomarkers and amyloid-PET allowed in vivo detection of amyloid pathology, atypical variants of Alzheimer's disease (AD) are increasingly recognized in clinical settings ${ }^{1-2}$. Autosomal dominant mutations in the amyloid precursor protein $(A P P)$, presenilin 1 (PSEN1), and presenilin 2 (PSEN2) genes also provide the highest level of diagnostic certainty ante-mortem ${ }^{1}$. Although mutations in the PSEN1 gene are found in less than $1 \%$ of AD patients, they are the most common cause of early-onset AD (EOAD), and a unique opportunity to understand disease mechanisms and their relationship to clinical phenotype ${ }^{3}$. More than 140 PSEN1 mutations have been identified so $\mathrm{far}^{3}$. PSEN1 mutations have been associated with the earliest $\mathrm{AD}$ onset, with an average age of $43^{4}$, and a typical amnestic syndrome of the hippocampal type ${ }^{5}$. Nevertheless, some PSEN1 mutations have been linked to atypical presentation, including behavioral and psychiatric symptoms ${ }^{5}$. In the present work, we describe an atypical late-onset presentation of the rare R377W PSEN1 mutation, and for the first time provide in vivo visualization of amyloid pathology in the proband as well as extensive characterization of her family.

\section{Patient consent}

The patient and her relatives provided informed consent for genetic analysis and for the use of their anonymized data for research purposes.

\section{Data Availability Statement}

The data that support the findings of this study are available in anonymized form upon reasonable request from the corresponding author. The data are not publicly available due to privacy or ethical restrictions. 


\section{Case report}

A 71-years old female Italian patient of Caucasian ethnicity was brought to the attention of our memory clinic for short-term episodic memory impairment. Her relatives reported troubles with memory subtly starting when she was 67 years old and increasing with time, accompanied by prominent personality changes, mood deflection, and apathy. On first clinical evaluation the patient made a childish impression, with disinhibition and inappropriateness in social interactions. She showed inflexibility, obsessive behavior, and reduced empathy. Her husband reported increased appetite for sweet foods and initial loss of autonomy. On neuropsychological assessment the episodic memory was clearly below normal limit, and showed cue-insensitivity on free and cued selective reminding test as in amnestic syndrome of the hippocampal type. The patient also had lower than normal scores on the domains of orientation in time, constructional apraxia, and on frontal assessment battery. Mini-mental state examination was 24/30. Beside higher functions, neurological examination was within normal limits. CSF analysis had been performed in another hospital: decreased 1-42 beta-amyloid $\left(410 \mathrm{pg} / \mathrm{mL}\right.$; cut-off $\left.>600^{6}\right)$, increased tau $(843 \mathrm{pg} / \mathrm{mL}$; cutoff $<400^{6}$ ), and tau/1-42 beta-amyloid ratio (2.06; cut-off $<0.52^{7}$ ) were compatible with AD. However, p-tau was not carried out to complete the CSF profile. Magnetic resonance imaging (MRI) showed mild parietal atrophy (Fig 1A-C). 18-Fluorodeoxyglucose positron emission tomography (FDG-PET) revealed bilateral hypometabolism more marked on the right hemisphere in the precuneus, posterior cingulum, inferior parietal lobes, temporal and frontal lobes (Fig 1D).

The family history of the patient was enriched with cases of early-onset dementia with mixed amnestic and behavioral/psychiatric features. The patient's older brother had a symptom onset at the age of 45 and a fatal outcome at the age of 54. The patient's younger sister is 69 years old and asymptomatic. The patient's father, paternal aunt and paternal grandfather had their symptom onset at the ages of 50, 45 and 65 years, and a disease course of 15, 5 and 10 years, respectively. The patient has two daughters aged 47 and 39 years, asymptomatic, who don't want to know their genetic status (Fig 2A).

Taking into account the recurrence of the disease in her family, an extensive genetic screening was carried out on the patient taking advantage of a next generation sequencing technique covering the spectrum of common and rare dementias ${ }^{8}$. This unraveled the presence of a heterozygous pathogenic mutation within the PSENI gene. This mutation consists of a thymine to adenosine 
base pair transition in exon $10^{9}$ resulting in the substitution of arginine to tryptophan $(\mathrm{g} 73211942$ A $>$ T NC_000014.9; c.1129A AGT>TGT NM_000021.4; $R 377 W$ ). The mutation was confirmed by direct sequencing (Fig 2B). Apolipoprotein E (ApoE) genotype was e3/e3. No mutations were found in chromosome 9 open reading frame 72 (c9orf72), progranulin (PRG), and microtubule associated protein tau $(M A P T)$ genes.

The patient underwent amyloid positron emission tomography (PET) with F18-florbetaben tracer, with both late image acquisitions for plaques detection and early image acquisitions as a surrogate index of regional blood perfusion ${ }^{10}$ (Fig $1 \mathrm{E}-\mathrm{G}$ ). The former revealed diffuse high to moderate density of cortical amyloid plaques, with the highest density in the frontal lobes, occipito-parietal regions, precunei and cingula (Fig 1E). The early image acquisition detected a reduced cortical blood perfusion in the lateral prefrontal regions, anterior temporal pole, posterior parietal lobes, and cingula (Fig 1F-G), consistent with hypometabolism on FDG-PET (Fig 1D).

In light of data reported by Borroni et al on R377W PSEN1 mutation ${ }^{11}$, the patient and her relatives were asked about epileptic episodes, which never occurred in the patient's life. The medical history was supported by the EEG of the patient, which did not disclose any pattern suspected for epilepsy or prion disease. 


\section{Discussion}

Here, we describe an atypical presentation of the rare R377W PSEN1 mutation, emphasizing the unusual late age of onset and the mixed frontotemporal and parieto-hippocampal dysfunction. In order to do so, beside a detailed clinical characterization, we provided a description of the family tree, conventional imaging findings and, for the first time, amyloid-PET.

The PSEN1 R377W mutation has previously been described only twice as probably pathogenic. The PSEN1 R377W mutation was first associated with EOAD starting at the age of 50 in a French patient who was not reported with atypical symptoms ${ }^{12}$. Two other members in his family were demented with unknown genotype. The second report described an Italian man diagnosed with epileptic seizures at the age of 60 and a prominent behavioural syndrome at the age of 66 , without affected relatives ${ }^{11}$.

The case here described presented with clinico-radiological features of AD (episodic memory deficit of the hippocampal type, disorientation, apraxia, hypometabolism in precunei and parietal lobes) paired with clinico-radiological features of behavioral FTD (disinhibition, apathy, lack of empathy, obsessivity, craving for sweets, frontotemporal hypometabolism), as in the behavioural variant of $\mathrm{AD}$ (bvAD) $)^{1-2,13-14}$. Here, we provide for the first time in vivo pathologic evidence of cortical amyloid plaque deposition linked to the PSEN1 R377W mutation. Remarkably, the highest plaque density was observed in the frontal lobes, as well as in the parietal lobes and precunei, consistently with the mixed disease phenotype. As a first difference with the other Italian case, the patient here presented did not show any language impairment, maybe consistently with the rightpredominant temporal pole involvement, which may also have contributed to the mixed behavioral and amnestic presentation ${ }^{11,15}$. Moreover, our patient did not have any indication for epileptic seizures, which were the first neurological manifestation and occurred early at the age of 60 in the other Italian patient ${ }^{11}$. Finally, the Italian male patient had no parietal abnormalities on brain imaging even on a 2 years follow-up ${ }^{11}$, while the patient here presented showed parietal atrophy at first MRI, as expected in bvAD ${ }^{2}$. To the best of our knowledge, the patient described in the present report is not a relative of the aforementioned Italian man. Nevertheless, the two patients lived in a close area, thus a founder effect could explain why this rare mutation has only been reported in individuals from Northern Italy and France ${ }^{16}$. 
In the present case, we showed how the age at disease onset and the disease duration can be strikingly different even within the same family, without anticipation and irrespectively of generation. It is possible that other genetic factors, such as the ApoE e3/e3 genotype, contributed to the late age at onset observed in the patient in contrast with other family members, as showed in large genetic studies on affected relatives ${ }^{17}$. The unusual late onset of our case leads us to speculate that, if more extensive screening was performed and not limited to younger cases, the prevalence of such "rare" mutations would be maybe found to be higher, at least in some geographical areas. The age at onset and the behavioral features of the present case would have made $c 9$ orf72 expansion, or GRN and MAPT mutations, the most obvious candidates for a targeted genetic investigation. On this note, the current indications on PSEN1 genetic testing stress the importance of an early disease onset ${ }^{18,19}$. On the contrary, we want to highlight the relevance of an extensive genetic screening, as with the use of next generation sequencing techniques, also in patients with late disease onset. Atypical presentations appear more and more to be the norm rather than the exception and targeting single genes on the basis of expected features such as the age of onset could leave significant gaps in our knowledge and in the management of patients. In this case report, we showed how a late age of onset should not be a reason to exclude patients from extensive AD genetic screening, especially in the presence of a positive family history. 


\section{Author contributions}

M.Sc., A.A.D., Y.A.L.P, E.S., and D.G. developed the study concept and design. M.Sc., A.A, C.F., F.S., M.Se., E.R., M.M., and G.M. were involved in data acquisition and analysis. M.Sc., A.A., and D.G. gave substantial contribution in drafting the manuscript and figures.

\section{Acknowledgements}

\section{Funding}

The European Academy of Neurology supported this work with a research fellowship granted to M. Scarioni.

\section{Disclosure of conflict of interest}

The authors report no conflicts of interest. 


\section{References}

1. Dubois B, Feldman HH, Jacova C, et al. Advancing research diagnostic criteria for Alzheimer's disease: the IWG-2 criteria. Lancet Neurol. 2014;13(6):614-629.

2. Ossenkoppele R, Pijnenburg YA, Perry DC, et al. The behavioural/dysexecutive variant of Alzheimer's disease: clinical, neuroimaging and pathological features. Brain. 2015;138(Pt 9):2732-2749.

3. Nikolac Perkovic M, Pivac N. Genetic Markers of Alzheimer's Disease. Adv Exp Med Biol. 2019;1192:27-52.

4. Dai MH, Zheng H, Zeng LD, Zhang Y. The genes associated with early-onset Alzheimer's disease. Oncotarget. 2017;9(19):15132-15143.

5. Ryan NS, Rossor MN. Correlating familial Alzheimer's disease gene mutations with clinical phenotype. Biomark Med. 2010;4(1):99-112.

6. Fumagalli GG, Sacchi L, Basilico P, et al. Monozygotic twins with frontotemporal dementia due to Thr272fs GRN mutation discordant for age at onset. J Alzheimers Dis. 2019;67(4):11731179 .

7. Duits FH, Teunissen CE, Bouwman FH, et al. The cerebrospinal fluid "Alzheimer profile": Easily said, but what does it mean?. Alzheimer's \& Dementia. 2014;10:713-723.e2.

8. Ghezzi L, Carandini T, Arighi A, et al. Evidence of CNS $\beta$-amyloid deposition in Nasu-Hakola disease due to the TREM2 Q33X mutation. Neurology. 2017;89(24):2503-2505.

9. PSEN-1. ALZFORUM. https://www.alzforum.org/mutations/psen-1. Accessed May 26, 2020.

10. Asghar M, Hinz R, Herholz K, Carter SF. Dual-phase [18F]florbetapir in frontotemporal dementia. Eur J Nucl Med Mol Imaging. 2019;46(2):304-311.

11. Borroni B, Pilotto A, Bonvicini C, et al. Atypical presentation of a novel Presenilin 1 R377W mutation: sporadic, late-onset Alzheimer disease with epilepsy and frontotemporal atrophy. Neurol Sci. 2012;33:375-378. 
12. Wallon D, Rousseau S, Rovelet-Lecrux A, et al. The French series of autosomal dominant early onset Alzheimer's disease cases: mutation spectrum and cerebrospinal fluid biomarkers. $J$ Alzheimers Dis. 2012;30(4):847-856.

13. Rascovsky K, Hodges JR, Knopman D, et al. Sensitivity of revised diagnostic criteria for the behavioural variant of frontotemporal dementia. Brain. 2011;134(Pt 9):2456-2477.

14. Singleton EH, Pijnenburg YA, Sudre CH, et al. Investigating the clinico-anatomical dissociation in the behavioral variant of Alzheimer's disease. MedRxiv. 2019. doi:10.1101/19006676.

15. Chan D, Anderson V, Pijnenburg Y, et al. The clinical profile of right temporal lobe atrophy. Brain. 2009;132(Pt 5):1287-1298.

16. Raveane A, Aneli S, Montinaro F, et al. Population structure of modern-day Italians reveals patterns of ancient and archaic ancestries in Southern Europe. Science Advances. 2019;5(9):eaaw3492.

17. Blacker D, Haines JL, Rodes L, et al. ApoE-4 and age at onset of Alzheimer's disease: the NIMH genetics initiative. Neurology. 1997; 48(1):139-47.

18. Bocchetta M, Mega A, Bernardi L, et al. Genetic Counseling and Testing for Alzheimer's Disease and Frontotemporal Lobar Degeneration: An Italian Consensus Protocol. J Alzheimers Dis. 2016;51(1):277-291.

19. Goldman JS, Van Deerlin VM. Alzheimer's Disease and Frontotemporal Dementia: The Current State of Genetics and Genetic Testing Since the Advent of Next-Generation Sequencing. Mol Diagn Ther. 2018;22(5):505-513. 

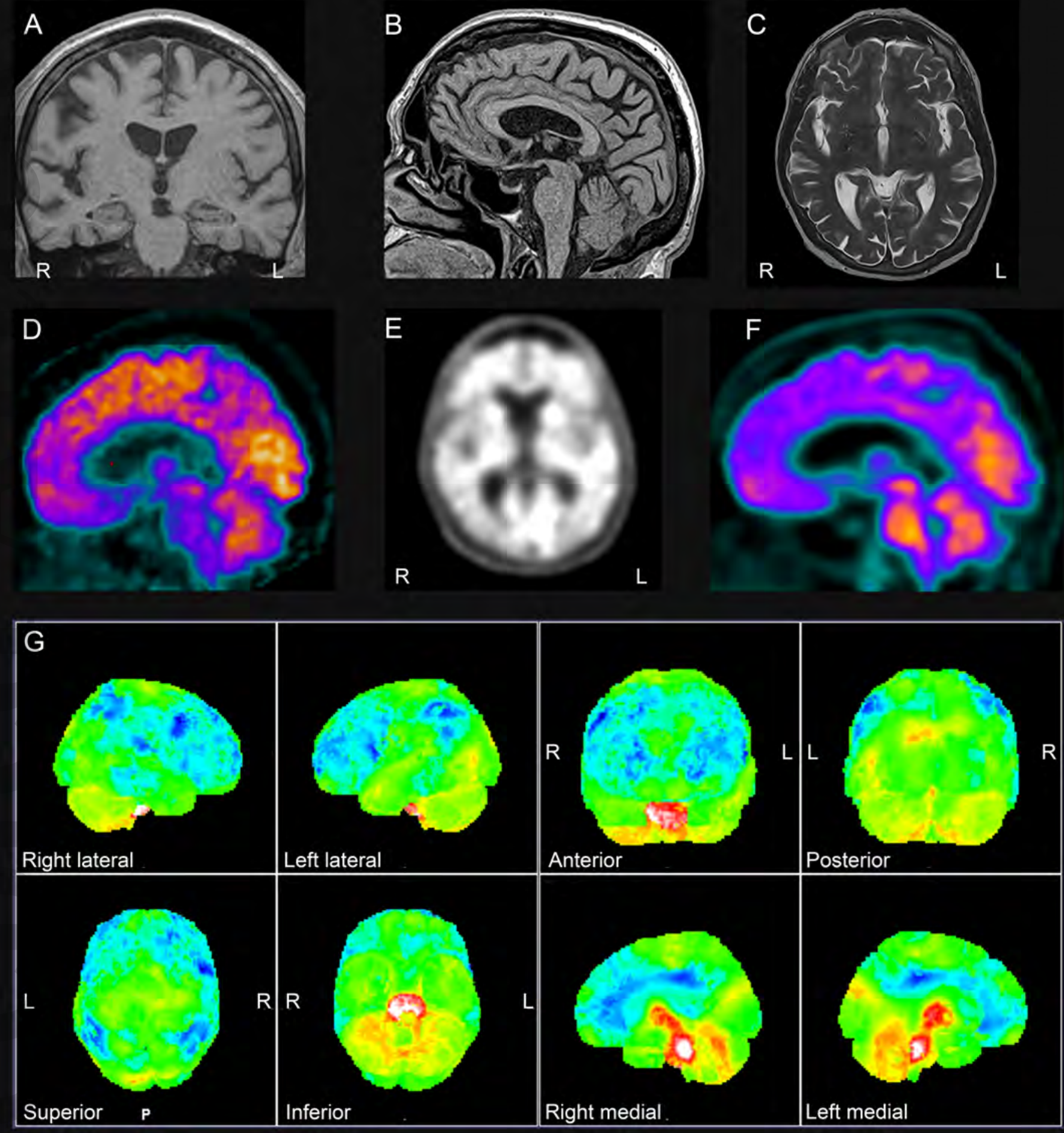

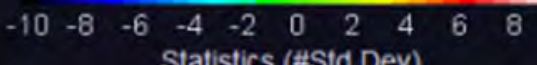

Statistics (\#SId Dev)

ene_14506_f1.tif

This article is protected by copyright. All rights reserved 

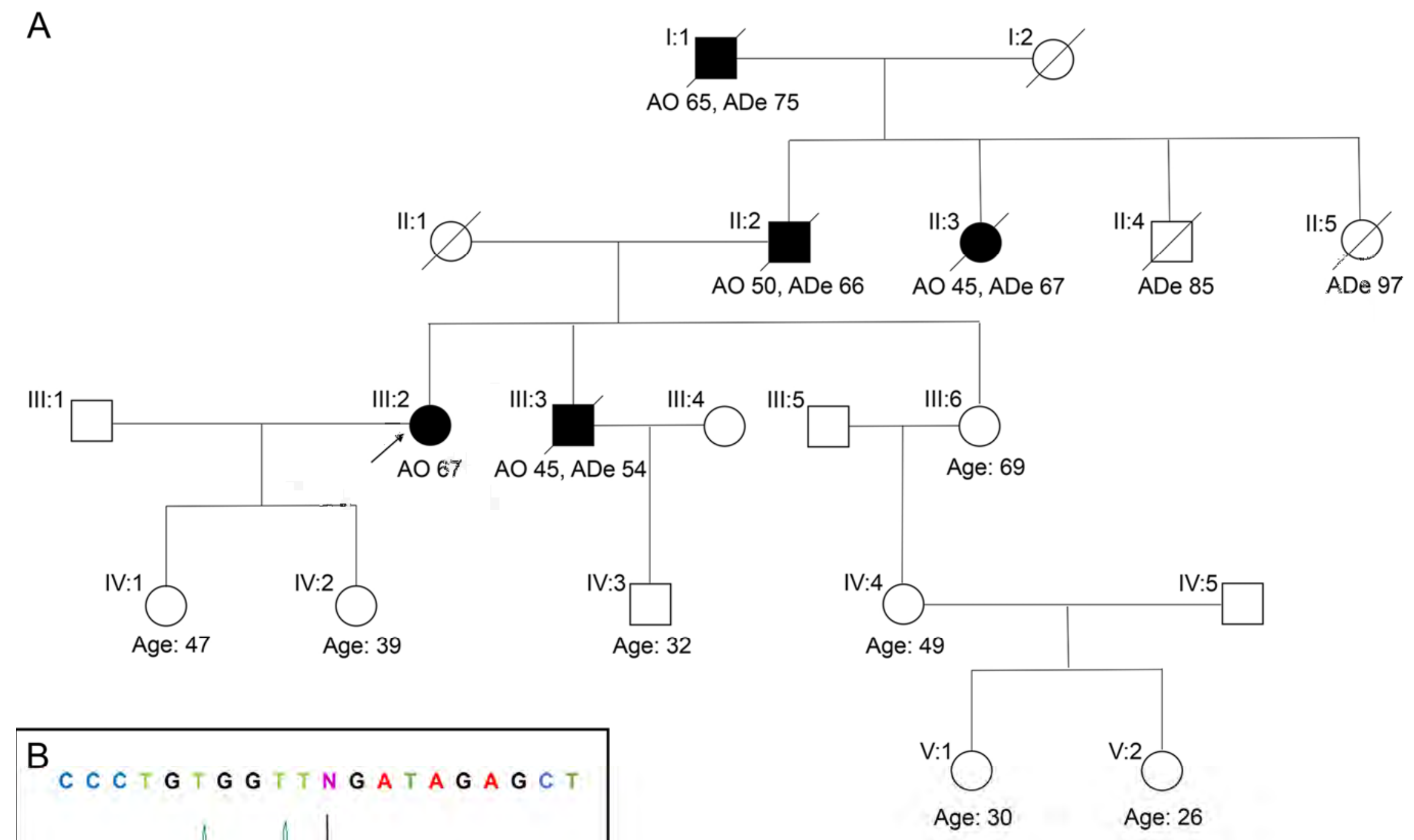


\section{THE MPORTANCE OF GREY AND WHITE MATTER In Multiple Sclerosis}

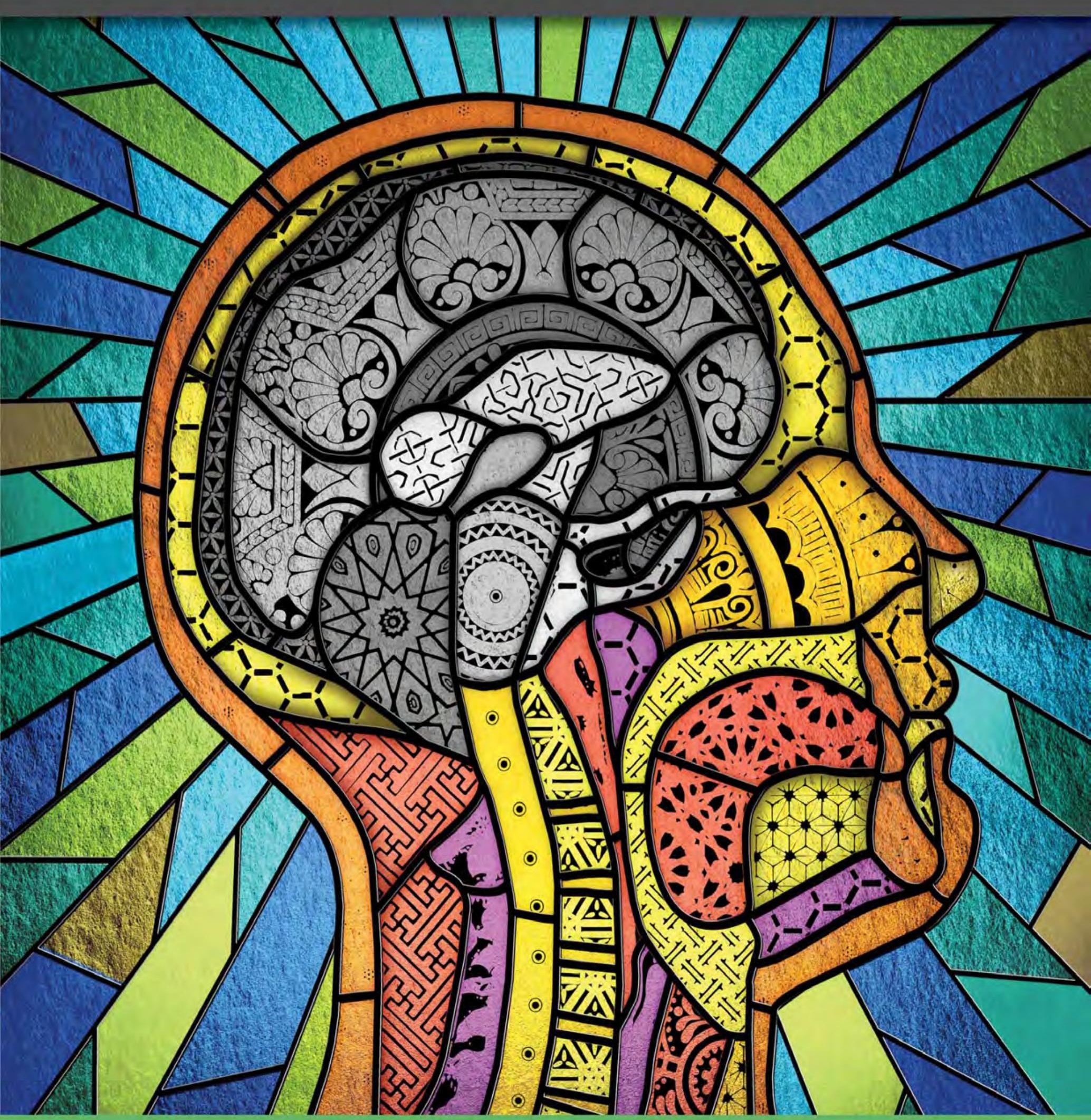

Visit GreyAndWhiteMS.com for more information. 